\title{
Revision to Reverse Total Shoulder Arthroplasty Restores Stability for Patients With Unstable Shoulder Prostheses
}

\author{
Nicholas M. Hernandez MD, Brian P. Chalmers MD, Eric R. Wagner MD, \\ John W. Sperling MD, Robert H. Cofield MD, Joaquin Sanchez-Sotelo MD, PhD
}

Received: 13 February 2017/ Accepted: 21 June 2017/Published online: 28 August 2017

(C) The Association of Bone and Joint Surgeons \& 2017

\begin{abstract}
Background Instability after shoulder arthroplasty remains a complication with limited salvage options. Reoperation for instability with anatomic designs has led to high rates of persistent instability, therefore we aimed to evaluate the use of RSA for treatment of prosthetic instability.

Questions/purposes (1) After revision shoulder arthroplasty to a reverse prosthesis (RSA), what is the
\end{abstract}

One of the authors certifies that he (JWS) or a member of his immediate family, has or may receive payments or benefits, during the study period, an amount of USD 10,000-USD 100,000, from Biomet Inc (Warsaw, IN, USA).

One of the authors certifies that he (RHC), or a member of his immediate family, has or may receive payments or benefits, during the study period, an amount of USD 100,001-USD 1,000,000, from Smith \& Nephew Inc (Andover, MA, USA), and an amount of USD 100,001-USD 1,000,000 from DJO Global (Austin, TX, USA). One of the authors certifies that he (JS-S), or a member of his immediate family, has or may receive payments or benefits, during the study period, an amount of USD 10,000-USD 100,000, from Stryker Corporation (Kalamazoo, MI, USA).

All ICMJE Conflict of Interest Forms for authors and Clinical Orthopaedics and Related Research ${ }^{\mathbb{R}}$ editors and board members are on file with the publication and can be viewed on request.

Clinical Orthopaedics and Related Research ${ }^{\mathbb{B}}$ neither advocates nor endorses the use of any treatment, drug, or device. Readers are encouraged to always seek additional information, including FDAapproval status, of any drug or device prior to clinical use.

Each author certifies that his institution approved or waived approval for the human protocol for this investigation and that all investigations were conducted in conformity with ethical principles of research.

N. M. Hernandez, B. P. Chalmers, E. R. Wagner, J. W. Sperling, R. H. Cofield, J. Sanchez-Sotelo ( $\square)$

Department of Orthopedic Surgery, Mayo Clinic, 200 First Street

SW, Rochester, MN 55905, USA

e-mail: sanchezsotelo.joaquin@mayo.edu survivorship free from dislocations at 2 and 5 years? (2) What factors are associated with dislocations? (3) What is the survivorship free from revision after revision to RSA? (4) From preoperation to postrevision to RSA, what are the clinical outcomes-the proportion of patients with moderate to severe pain, shoulder elevation and external rotation ROM, American Shoulder and Elbow Surgeons scores, and Simple Shoulder Test scores?

Methods All shoulder arthroplasties revised for prosthetic instability using RSA components between January 2004 and July 2014 were retrospectively studied. During the period in question, we performed 82 revisions for instability of an anatomic total shoulder arthroplasty (TSA) $(\mathrm{n}=$ $62)$, hemiarthroplasty $(n=13)$, or reverse TSA $(n=7)$. We typically used a reverse TSA to treat this problem, but we identified 12 treated in other ways, including revision of a TSA to hemiarthroplasty $(n=3)$, revision of a reverse TSA to hemiarthroplasty $(\mathrm{n}=2)$, revision of hemiarthroplasty to a hemiarthroplasty $(\mathrm{n}=1)$, and revision of an anatomic TSA to another anatomic TSA $(n=6)$. This left 70 patients for evaluation; of those, $65(93 \%)$ were available for analysis at a mean of 3 years (range, 2-10 years). A total of seven patients died. Eight of the 65 shoulders were not evaluated during the last 5 years, including three in patients who died earlier. The mean age of the patients at the time of revision RSA was 65 years (range, $40-89$ years). Data were obtained from a longitudinally maintained institutional joint registry. Instability was defined as severe subluxation confirmed on clinical and radiographic examinations. We evaluated pain and ROM, and Kaplan-Meier curves were used to estimate survivorship.

Results The survivorship free from dislocation at 2 and 5 years was $87 \%$ (95\% CI, 80\%-94\%) and 79\% (95\% CI, $67 \%-91 \%)$ respectively, with 10 of $65(15 \%)$ patients having an episode of dislocation after revision surgery. 
Persistent instability was more common in those with a BMI greater than $35 \mathrm{~kg} / \mathrm{m}^{2}$ (hazard ratio [HR], 5; 95\% CI, $2-16 ; \mathrm{p}=0.008)$ and prior hemiarthroplasty (HR, 5; 95\% CI, 2-16; $\mathrm{p}=0.005)$, whereas patients who had undergone a previous TSA were less likely to have persistent instability (HR, 0.08; 95\% CI, 0.0-0.30; p $<0.001$ ) The survival free from rerevision for any indication at 2 and 5 years was $85 \%(95 \% \mathrm{CI}, 76 \%-94 \%)$ and $78 \%(95 \% \mathrm{CI}$, $66 \%-90 \%$ ) respectively; with the numbers available, we were not able to find associated factors. Fewer patients had moderate or severe pain after revision to RSA (preoperative: 48 of 65 [74\%]; postoperative: nine of 65 [14\%]; p < 0.001). After surgery, patients showed improvement in shoulder elevation (preoperative: $42^{\circ}\left[ \pm 30^{\circ}\right.$, postoperative: $112^{\circ}\left[42^{\circ}\right]$; mean difference, $70^{\circ}\left[95 \% \mathrm{CI},-83^{\circ}\right.$ to $\left.57^{\circ}\right]$; $<<0.001$ ) and external rotation (preoperative: $20^{\circ}[ \pm$ $\left.22^{\circ}\right]$, postoperative: $42^{\circ}\left[ \pm 23^{\circ}\right]$; mean difference, $22^{\circ}$ $\left[95 \% \mathrm{CI},-30^{\circ}\right.$ to $\left.\left.-14^{\circ}\right] ; \mathrm{p}<0.001\right)$. American Shoulder and Elbow Surgeons scores improved (preoperative: 21 [ 10], postoperative: $68[ \pm 14]$, mean difference, $46[95 \%$ CI, -58 to -35$]$; $\mathrm{p}<0.001$ ); where a higher score is better. Simple Shoulder Test scores also improved (preoperative: $2 / 12[ \pm 2]$, postoperative: 7/12 [ \pm 3$]$; mean difference, 5 [95\% CI, -7 to -2.17 ]; $\mathrm{p}<0.001$ ); where a higher score is better.

Conclusions Revision RSA for prosthetic instability after shoulder arthroplasty is associated with reasonable implant survival and few complications. Approximately one in seven patients will have a recurrent dislocation. In patients with persistent instability or with risk factors for instability, consideration should be given for use of larger glenospheres and increasing the lateral offset at the time of RSA. Level of Evidence Level IV, therapeutic study.

\section{Introduction}

The risk of instability after anatomic shoulder arthroplasty has been estimated to be approximately 5.2\% [7]. Wide ranges of instability after primary reverse shoulder arthroplasty (RSA) also have been reported $(2.4 \%-31 \%)$ [4-6, 8, 16, 20]. Treatment options for an unstable shoulder arthroplasty include nonoperative management or operatively addressing factors leading to instability [13], including open reduction and soft tissue procedures, revision to hemiarthroplasty [9] or RSA [18], or resection arthroplasty [11]. Sanchez-Sotelo et al. [13] reported a high risk of persistent pain and instability when revision surgery was performed before the advent of RSA. They found that addressing soft tissue laxity and component malposition only had modest results, with more than half of the patients having remaining instability [13]. Abdel et al. [1] reported promising results with revision RSA for an unstable anatomic shoulder arthroplasty with few instances of recurrent instability.

RSA has emerged as an attractive alternative for salvage in various shoulder conditions. Initially developed for surgical treatment of cuff tear arthropathy, the indications for RSA have expanded, and it may be an option for patients with proximal humerus fractures [12], rheumatoid arthritis [13], fixed glenohumeral dislocation, tumor surgery, fracture nonunion, glenoid bone loss, and revision arthroplasty [14, 17]. In the setting of recurrent dislocations after shoulder replacement, the semiconstrained nature of RSA and the possibility of relative lengthening of the arm and variable offsets depending on the sizes of glenospheres and humeral bearings used make revision RSA especially attractive for an unstable shoulder arthroplasty [3]. Reoperation for instability with anatomic designs had led to high rates of persistent instability. RSA may offer superior outcomes but still has its own limitations.

We therefore asked: (1) After revision shoulder arthroplasty to RSA, what is the survivorship free from dislocations at 2 and 5 years? (2) What factors are associated with dislocations? (3) What is the survivorship free from revision after revision to RSA? (4) From preoperation to postrevision to RSA, what are the clinical outcomesthe proportion of patients with moderate to severe pain, shoulder elevation and external rotation ROM, American Shoulder and Elbow Surgeons (ASES) scores, and Simple Shoulder Test (SST) scores?

\section{Materials and Methods}

After approval from our institutional review board for our minimal-risk study, our department total joint registry database was used to identify all patients who underwent revision to a RSA between January 2004 and July 2014. Review of the medical records was used to identify all revisions performed for prosthetic instability. This was a retrospective study drawn from a longitudinally maintained institutional database, which contains patient demographics, details of the surgery, medical and surgical history, complications, and clinical outcome information.

During the study period, 82 revisions were performed for instability of an anatomic total shoulder arthroplasty (TSA) $(n=62)$, hemiarthroplasty $(n=13)$, or reverse TSA $(n=7)$. We typically used a reverse TSA to treat this problem, but we identified 12 that were treated in other ways, including revision of a TSA to hemiarthroplasty $(\mathrm{n}=$ $3)$, revision of a reverse TSA to hemiarthroplasty $(n=2)$, revision of hemiarthroplasty to a hemiarthroplasty $(n=1)$, and revision of an anatomic TSA to another anatomic TSA $(n=6)$. This left 70 patients for evaluation; of those, 65 (93\%) were available for analysis at a mean of 3 years 
(range, 2-10 years). A total of seven patients died. Eight of the 65 shoulders were not evaluated during the last 5 years, including three in patients who died earlier. Unstable implants revised included hemiarthroplasty in 12 shoulders (18\%), anatomic TSA in 48 shoulders (74\%), and RSA in five shoulders $(8 \%)$.

Patients were followed until dislocation, rerevision, reoperation, or until final clinical followup at a minimum of 2 years.

We defined instability as dislocation or severe subluxation confirmed on clinical and radiographic examinations. Acute dislocations were attempted to be relocated, but all patients in this cohort had recurrent instability develop, and chose to undergo revision with RSA. Rerevision was defined as revision of components in place. Reoperation was defined as additional surgery resulting in no exchange of components in place but could include exchange of modular polyethylene. Thirtynine patients were women $(60 \%)$ with a mean age of 65 years (range, 40-89 years) and a mean BMI of $32 \mathrm{~kg} / \mathrm{m}^{2}$ (range, $1-57 \mathrm{~kg} / \mathrm{m}^{2}$ ).

\section{Surgical Technique}

All patients underwent revision RSA at our institution by one of five fellowship trained shoulder surgeons (RHC, BTE, JSS, JWS, SPS). Patients were included if they had pain and clinical appearance of a dislocated shoulder that was confirmed on radiographic review. Implants were used from three different companies, including 15 Encore Reverse ${ }^{\circledR}$ Shoulder Prostheses (DJO Global, Austin, TX, USA), three Delta III and four Delta Xtend ${ }^{\mathrm{TM}}$ (DePuy Orthopaedics, Warsaw, IN, USA), and 40 Comprehensive ${ }^{\circledR}$ Reverse Shoulder prostheses (Biomet, Warsaw, IN, USA). Five patients underwent glenoid bone augmentation; allograft was used in three patients and autograft from the cut surface of the proximal humerus in two patients. We used allograft reconstruction of the proximal humerus in two patients (allograft-prosthetic composite). Postoperatively patients wore a shoulder abduction brace or a sling. For the first 2 to 3 weeks patients worked on wrist and elbow ROM. By 6 weeks patients were allowed to work on ROM in all directions and they no longer had to wear the brace or sling. By 12 weeks patients were allowed to perform strengthening exercises.

Preoperative and postoperative pain and active shoulder motion were collected for all patients. Pain levels were graded as none, mild, moderate with activities, moderate at rest, or severe. Shoulder ROM was assessed using goniometers. ASES and SST scores were evaluated preoperative and postoperatively. Radiographs were evaluated before surgery to confirm the presence of instability and at the most recent followup for evidence of loosening, persistent instability, or other radiographic complications.

\section{Statistical Analysis}

The differences between preoperative and postoperative data were compared using the paired t-test for continuous variables and Fisher's tests for dichotomous variables. Kaplan-Meier survivorship curves were constructed for survivorship free of dislocation, implant survivorship free of revision, and implant survivorship free of revision or reoperation for any indication. We analyzed primary and secondary endpoints of revision surgery, dislocations, and clinical outcomes.

\section{Results}

The survivorship free from dislocation at 2 and 5 years was $87 \%$ (95\% CI, 80\%-94\%) and 79\% (95\% CI, 67\%-91\%), respectively (Fig. 1); overall, dislocations in revision RSAs occurred in 10 of 65 patients $(15 \%)$. Of the 10 patients (15\%) who had recurrent instability develop, eight had anterior dislocations, with six of these eight being noted to have subscapularis failure. Two patients had anterosuperior instability, with one of the two having massive rotator cuff tearing and the other having subscapularis failure. Of the patients with unstable hemiarthroplasties at the time of revision to RSA, two had proximal humerus bone loss treated with bone graft, two had tuberosity resorption, three had massive rotator cuff tears, three had glenoid bone loss treated with bone graft, and six had a diminished or absent subscapularis. Of the four patients who had dislocations but did not have reoperation, one was treated with closed

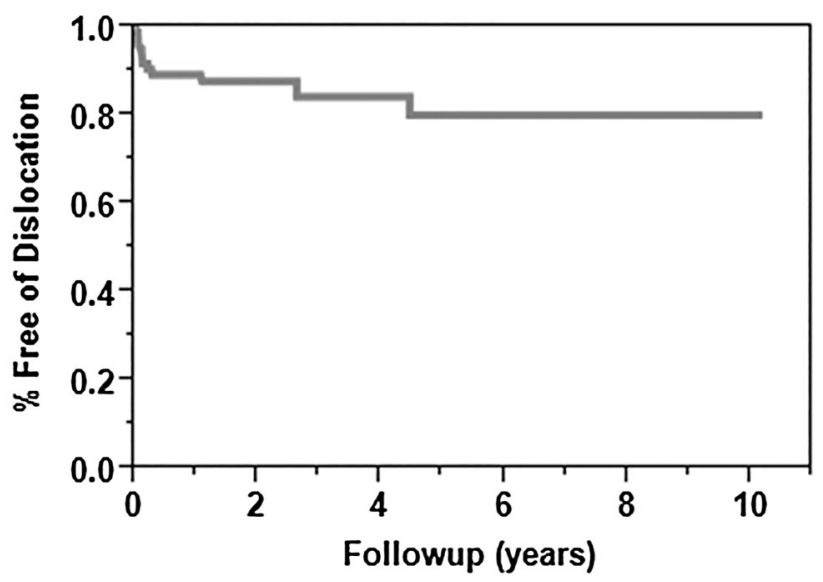

Fig. 1 The Kaplan-Meier survival curve shows the survival free of dislocation at 2 and 5 years was $87 \%$ (95\% CI, 80\%-94\%) and $79 \%$ (95\% CI, 67\%-91\%), respectively. 
Table 1. Hazard ratios for dislocation

\begin{tabular}{llc}
\hline Risk factor & Hazard ratio (95\% CI) & p value \\
\hline TSA & $0.08(0.01-0.30)$ & $<0.001^{*}$ \\
Hemiarthroplasty & $5.43(1.74-16.37)$ & $0.005^{*}$ \\
BMI $>35 \mathrm{~kg} / \mathrm{m}^{2}$ & $4.71(1.50-15.98)$ & $0.009^{*}$ \\
RSA & $2.97(0.89-9.02)$ & 0.070 \\
Greater than 3 prior surgeries & $3.21(0.72-10.54)$ & 0.110 \\
Rheumatoid arthritis & $0.00(1.70-1.70)$ & 0.130 \\
Prior proximal humerus fracture & $2.48(0.73-7.80)$ & 0.140 \\
Laborer & $0.00(2.59-2.59)$ & 0.210 \\
Smoker & $1.91(0.43-6.30)$ & 0.360 \\
Gender (male) & $1.35(0.39-4.49)$ & 0.620 \\
Diabetes mellitus & $1.01(0.15-3.94)$ & 0.990 \\
\hline
\end{tabular}

TSA $=$ total shoulder arthroplasty; RSA $=$ reverse shoulder arthroplasty; * significant $\mathrm{p}$ value.

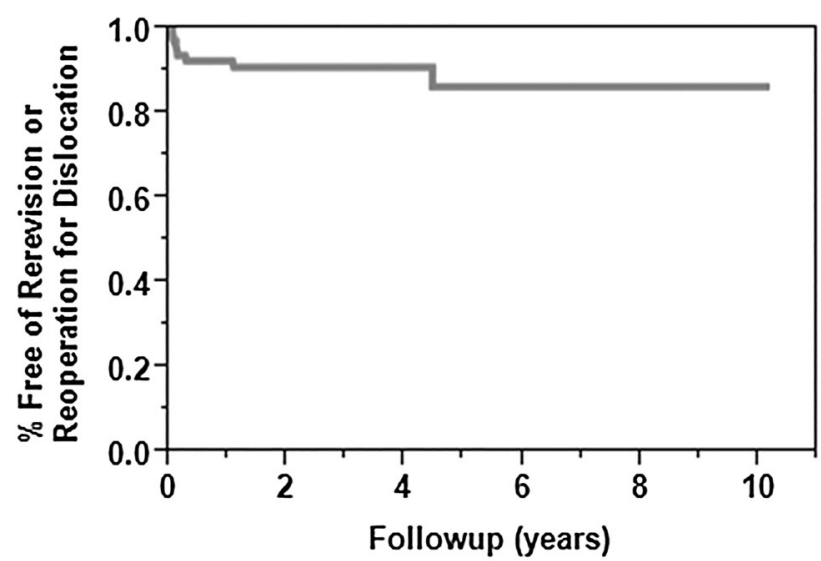

Fig. 2 The Kaplan-Meier survival curve shows the survival free of reoperation or rerevision for dislocation at 2 and 5 years was $94 \%$ (95\% CI, 88\%-100\%) and 86\% (95\% CI, 75\%-97\%), respectively.

reduction and the dislocation remained reduced. Three patients had chronically dislocated shoulders. In the three patients with chronic dislocations, one patient felt use of the shoulder was adequate and chose to have no further treatment, the second patient had two closed reductions, and both of these patients had dislocations until death. The third patient with a dislocation elected to pursue no additional treatment given the complexity of her problem.

Dislocation was more common in patients with a BMI greater than $35 \mathrm{~kg} / \mathrm{m}^{2}$ (hazard ratio [HR], 5; 95\% CI, 2-16; $\mathrm{p}=0.008)$ and prior hemiarthroplasty (HR, 5 ; 95\% CI, $2-$ $16 ; \mathrm{p}=0.005)$. Dislocation was less common in patients with a prior TSA (HR, 0.08 ; 95\% CI, 0.01-0.30; $\mathrm{p}<0.001$ ) (Table 1). Six of $10(60 \%)$ patients who experienced a dislocation had a reoperation or repeat revision surgery to address their dislocation. Our intent to treat was nine patients, as one patient underwent closed reduction of the
Table 2. Hazard ratios for repeat revision or reoperation for dislocation

\begin{tabular}{llc}
\hline Risk factor & Hazard ratio $(95 \% \mathrm{CI})$ & $\mathrm{p}$ value \\
\hline TSA & $0.14(0.02-0.57)$ & $<0.005^{*}$ \\
$\mathrm{BMI}>35 \mathrm{~kg} / \mathrm{m}^{2}$ & $4.06(1.07-16.51)$ & $0.040^{*}$ \\
$\mathrm{RSA}$ & $3.88(0.94-14.94)$ & 0.060 \\
Hemiarthroplasty & $2.77(0.58-10.49)$ & 0.180 \\
Rheumatoid arthritis & $0.00(2.31-2.31)$ & 0.180 \\
Laborer & $0.00(3.50-3.50)$ & 0.270 \\
Gender (male) & $2.11(0.47-10.75)$ & 0.320 \\
Prior proximal humerus fracture & $1.94(0.40-7.95)$ & 0.380 \\
Diabetes mellitus & $1.49(0.22-6.48)$ & 0.640 \\
Smoker & $0.85(0.05-4.68)$ & 0.880 \\
Greater than 3 prior surgeries & $1.11(0.06-6.07)$ & 0.920 \\
\hline
\end{tabular}

TSA = total shoulder arthroplasty; $\mathrm{RSA}=$ reverse shoulder arthroplasty; * significant $\mathrm{p}$ value.

dislocation which then remained reduced, and three patients chose to have no additional treatment for the dislocations. The survival free from reoperation or rerevision for dislocation at 2 and 5 years was $94 \%$ (95\% CI, 88\%$100 \%$ ) and $86 \%$ (95\% CI, 75\%-97\%) (Fig. 2). Rerevision or reoperation for dislocation was more common in those with a BMI greater than $35 \mathrm{~kg} / \mathrm{m}^{2}$ (HR, 4; 95\% CI, 1-17; p $=0.040)$ and was less common in those with prior TSAs (HR, 0.14; 95\% CI, 0.02-1; $\mathrm{p}=0.005$ ) (Table 2). Of the six patients who had a rerevision or reoperation for dislocation, two underwent a salvage hemiarthroplasty because they did not have adequate soft tissue tensioning to support a repeat revision RSA, and both then had chronic dislocations. One patient with a chronically dislocated hemiarthroplasty has substantial disability in her operative extremity and the second patient with a dislocated hemiarthroplasty has had intermittent pain. Both have elected to pursue no further treatment. One patient had polyethylene exchange to a retentive type and had no additional instability. Three patients had a larger glenosphere placed for instability and have had no further episodes of dislocation. One of the patients whose glenosphere was revised also underwent revision of a loose humeral component.

The survival free from rerevision for any indication at 2 and 5 years was $85 \%(95 \% \mathrm{CI}, 76 \%-94 \%)$ and $78 \%(95 \%$ CI, 66\%-90\%) respectively (Fig. 3). Overall, 12 (18\%) shoulders underwent repeat revision surgery at a mean of 1.5 years (range, 0.05-6 years) postoperatively. Four shoulders $(6 \%)$ had glenoid component loosening develop and underwent revision surgery to hemiarthroplasties. One shoulder had loosening of the humeral component 6 years after revision RSA and underwent humeral component repeat revision. Two patients sustained periprosthetic fractures; one had a loose humeral stem treated with a 
proximal humeral replacement, whereas the other was treated with open reduction, internal fixation, and retention of the implant. No risk factors were identified for repeat revision for any indication (Table 3).

Fewer patients had moderate or severe pain after undergoing revision surgery to an RSA (preoperative: 48 of

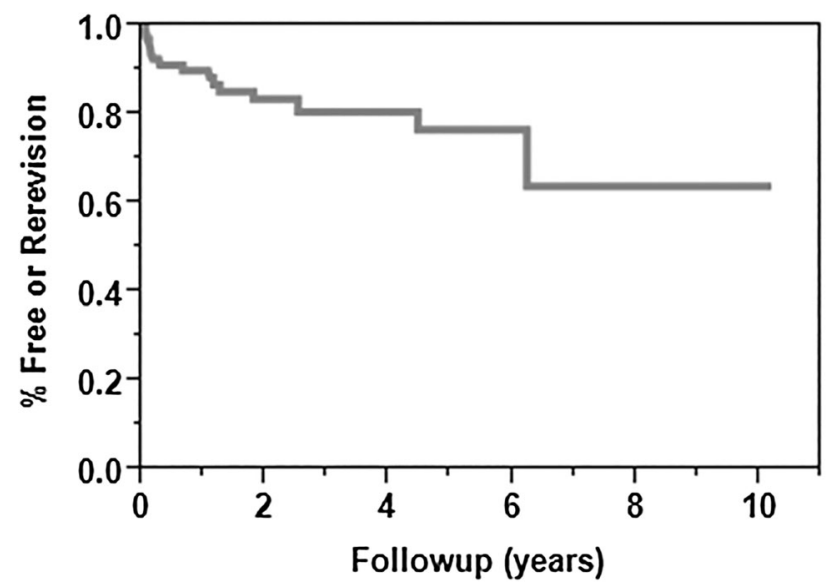

Fig. 3 The Kaplan-Meier survival curve shows the survival free of rerevision at 2 and 5 years was $85 \%(95 \% \mathrm{CI}, 76 \%-94 \%)$ and $78 \%$ (95\% CI, 66\%-90\%), respectively.

Table 3. Hazard ratios for repeat revision

\begin{tabular}{lll}
\hline Risk factor & Hazard ratio (95\% CI) & p value \\
\hline RSA & $2.73(0.84-7.77)$ & 0.090 \\
TSA & $0.43(0.15-1.18)$ & 0.100 \\
BMI $>35 \mathrm{~kg} / \mathrm{m}^{2}$ & $2.30(0.77-6.44)$ & 0.130 \\
Laborer & $0.00(1.79-1.79)$ & 0.140 \\
Diabetes mellitus & $1.88(0.52-5.63)$ & 0.310 \\
Greater than 3 prior surgeries & $0.53(0.03-2.72)$ & 0.510 \\
Prior proximal humerus fracture & $1.45(0.40-4.36)$ & 0.540 \\
Rheumatoid arthritis & $0.63(0.03-3.16)$ & 0.630 \\
Gender (male) & $1.28(0.42-3.73)$ & 0.650 \\
Hemiarthroplasty & $1.23(0.28-3.87)$ & 0.750 \\
Smoker & $1.04(0.16-3.79)$ & 0.960 \\
\hline
\end{tabular}

TSA = total shoulder arthroplasty; $\mathrm{RSA}=$ reverse shoulder arthroplasty.
65 [74\%], postoperative: nine of 65 [14\%]; $\mathrm{p}<0.001)$. After surgery, patients showed improvement in shoulder elevation (preoperative: $42^{\circ}\left[ \pm 30^{\circ}\right]$, postoperative: $112^{\circ}$ [ $\pm 42^{\circ}$ ]; mean difference, $70^{\circ}$; $95 \% \mathrm{CI},-83^{\circ}$ to $57^{\circ}$; $\mathrm{p}<$ 0.001 ) and external rotation (preoperative: $20^{\circ}\left[ \pm 22^{\circ}\right]$, postoperative: $42^{\circ}\left[ \pm 23^{\circ}\right.$ ]; mean difference, $22^{\circ} ; 95 \% \mathrm{CI}$, $-30^{\circ}$ to $\left.-14^{\circ} ; \mathrm{p}<0.001\right)$. The ASES scores improved (preoperative: $21[ \pm 10]$, postoperative: $68[ \pm 14]$; mean difference, 46 ; $95 \% \mathrm{CI},-58$ to $-35 ; \mathrm{p}<0.001$ ), as did the Simple Shoulder Test scores (preoperative: $2[ \pm 2]$, postoperative: 7 [ \pm 3 ]; mean difference, $5 ; 95 \% \mathrm{CI},-7$ to $-2 ; \mathrm{p}<0.001)($ Table 4$)$.

\section{Discussion}

Instability after shoulder arthroplasty is recognized as a difficult complication to resolve, and often is multifactorial [13]. Before the introduction of RSA, most shoulder arthroplasties revised for instability were treated with a combination of component exchange and soft tissue rebalancing. Unfortunately, despite efforts to improve component position and to reconstruct or augment the shoulder capsule and rotator cuff, sometimes with transfers, the risk of persistent or recurrent instability reported was extremely high, up to $58 \%$ in one series [13]. Increased indications for RSA led to some surgeons considering revision to RSA for patients with unstable anatomic shoulder prostheses [1]. Because of high failure rates of revision to anatomic components, we aimed to report redislocation rates and outcomes after revision to RSA.

The results of this study should be interpreted in light of certain limitations. We acknowledge that this is a retrospective study and lacks a control group regardless of the type of primary arthroplasty. During the same time this study was conducted, six patients underwent revision of the shoulder arthroplasty to hemiarthroplasty and six underwent revision of the shoulder arthroplasty to TSA for unstable prostheses. The decision to use RSA introduces a selection bias. When there was an absent subscapularis it was felt that RSA would continue to be unstable and it

Table 4. Clinical outcomes*

\begin{tabular}{|c|c|c|c|c|}
\hline Parameter & Preoperative & Postoperative & Odds ratio and mean difference $[95 \%$ CI ] & $\mathrm{p}$ value \\
\hline Moderate or severe pain & $48 / 65(\%)$ & $9 / 65(\%)$ & $0.06(0.02-0.1)$ & $\mathrm{p}<0.001$ \\
\hline Elevation ROM & $42( \pm 30.44)$ & $112( \pm 42.15)$ & $70(82.76-57.24)$ & $\mathrm{p}<0.001$ \\
\hline External Rotation ROM & $20( \pm 21.65)$ & $42( \pm 23.15)$ & $22(-29.8$ to 14.2$)$ & $\mathrm{p}<0.001$ \\
\hline ASES & $21.4( \pm 9.97)$ & $67.7( \pm 14.16)$ & $46.3(-57.6$ to 34.9$)$ & $\mathrm{p}<0.001$ \\
\hline SST & $2.3( \pm 1.82)$ & $7.0( \pm 2.96)$ & $4.7(-7.3$ to 2.2$)$ & $\mathrm{p}<0.001$ \\
\hline
\end{tabular}

* There was improvement in pre- to postoperative pain, shoulder elevation, and external rotation $(\mathrm{p}<0.01)$; ASES = American Shoulder and Elbow Surgeons; SST = Simple Shoulder Test. 
might have been used less frequently. In addition, during the early study period, RSA was becoming more common for treatment of unstable prostheses, and some surgeons may have felt less comfortable with RSA for this indication which might have biased the choice of implant. All surgeries were performed at a single academic center. Clinical followup of 2 years was adequate for early results, but further followup is needed to see how these patients perform long term. Further, five of 65 patients were not seen during the last 5 years and three other patients died before 5 years followup. These patients could have had additional instability events. The surgeons in this series were highly experienced in dealing with complex revision shoulder arthroplasty. Therefore the results in this study likely present a best-case scenario and may underestimate the true rate of recurrent instability. Finally, all patients had preoperative prosthetic instability and underwent revision of the prosthesis to the reverse prosthesis, however, different prostheses (TSA, hemiarthroplasty, and RSA prostheses) were included. We attempted to identify specific risk factors, including type of prior prosthesis, for poorer outcomes postoperatively.

The results of our study confirm the relatively high rate of restoration of shoulder stability with RSA components used for treatment of prosthetic instability. Other studies have provided some information regarding this topic. In a study by Boileau et al. [3], two of the 19 patients who had a revision RSA had redislocation (11\%). In another study of 31 revision RSAs, including six for instability, Patel et al. [12] reported patients experienced good pain relief and functional outcomes, with one (3\%) recurrent dislocation. Walker et al. [18] analyzed patients who underwent revision RSA for failed TSA, 19 of whom underwent revision surgery for instability. Similar to the current study, they noted improvement in pain and shoulder function; they had a $10 \%$ redislocation rate. One $(5 \%)$ patient experienced dislocation 5 weeks after surgery and was treated with closed reduction, and one (5\%) patient underwent rerevision surgery with implantation of a long-stemmed prosthesis for humeral and glenoid-sided loosening. Abdel et al. [1] reported 33 unstable anatomic shoulder arthroplasties converted to RSAs. Their patients had a mean 42month followup, and at latest followup 31 (94\%) shoulders were stable. Sanchez-Sotelo et al. [13] found a very high rate of instability (58\%) in patients treated with non-RSA solutions that focused on improving component position and soft tissue tension. In the current study, we estimated the 5-year survivorship free of dislocation at $79 \%$ and 5year survivorship free of rerevision or reoperation for dislocation at $86 \%$, which we believe is an understandable rate considering the challenging problem and underlying disorder. It also is a substantial improvement compared with addressing instability with non-RSA solutions [13].
A BMI greater than $35 \mathrm{~kg} / \mathrm{m}^{2}$ was found to be a risk factor for dislocation. Werner et al. [19] reported that dislocation was increased 1 year postoperatively in patients who were superobese $(2.9 \%)$ compared with patients who were not obese $(1.7 \%)$ who were treated with primary TSA or RSA $(\mathrm{p}<0.05)$. Patients who are obese can be counseled that there may be an increased risk for recurrent instability after revision RSA. Furthermore, patients with an unstable hemiarthroplasty before revision RSA were found to have higher rates of dislocation (HR, 5; 95\% CI, $2-16 ; p=0.005)$. On the contrary, revision RSA for an unstable anatomic prosthesis was highly successful with a low rate of postoperative recurrent dislocation [18]. Patients with a hemiarthroplasty may have structural changes including increased bone loss that may not be present to the same degree in patients with a TSA, which may place them at increased risk of dislocation after revision RSA.

The survival free from rerevision for any indication was found to be acceptable in this series. Unlike other studies, we did not identify any risk factors for rerevision of RSA, but similar to some studies, a common indication for revision was instability. Boileau et al. [2] evaluated revision of failed RSAs and the most-common indications were prosthetic instability (48\%) and humeral loosening, derotation, or fracture (21\%). At a mean of 34 months followup, 32 patients $(86 \%)$ required no additional revision, three patients (8\%) underwent resection arthroplasty, and two (6\%) underwent conversion of the RSA to hemiarthroplasty. Holcomb et al. [10] reported that of 14 patients with RSA glenoid baseplate failure undergoing revision to RSA, two of $14(14 \%)$ had repeat baseplate failure requiring repeat revision. Stephens et al. [15] reported that during a 12 -year period $6 \%$ of RSAs performed required revision. Those who underwent index RSA for failed hemiarthroplasty had the highest revision rate $(10 \%)$. Revision was performed for baseplate failure (2.5\%), infection (1.3\%), humeral dissociation $(0.7 \%)$, glenosphere dissociation $(0.6 \%)$, glenohumeral dislocation $(0.4 \%)$ periprosthetic fracture $(0.4 \%)$, and aseptic humeral loosening $(0.3 \%)$. The results of our study are important because no other studies, to our knowledge, have evaluated rerevision RSA in the context of prosthetic instability.

The majority of patients in our study had substantial improvement in pain, ROM, and shoulder outcome scores. These results represent an improvement from non-RSA treatment options for instability. Sanchez-Sotelo et al. [13] reported that 23 of $33(70 \%)$ patients had unsatisfactory results, using the Neer rating system, after soft tissue tensioning and component reorientation to treat unstable shoulder arthroplasties. Abdel et al. [1] found, using the Neer rating system, only 10 of $33(30 \%)$ had unsatisfactory results, whereas 10 of $33(30 \%)$ and 13 of 33 
$(40 \%)$ reported satisfactory or excellent results, respectively, when converting unstable anatomic shoulder arthroplasties to RSAs. Walker et al. [18] evaluated revision to RSA for failed TSA with the most-common reason being glenohumeral instability. They found that the ASES score improved from 38.5 preoperatively to 67.5 postoperatively and the median SST score improved from 1 to 5 . Abduction improved from $45^{\circ}$ to $100^{\circ}$ and external rotation improved from $12.5^{\circ}$ to $49.5^{\circ}$. Revision to RSA provides more predictable clinical improvements for unstable prostheses compared with anatomic surgical techniques.

At 5 years, four of five patients will be free of a recurrent dislocation, whereas approximately one of 10 will undergo a procedure for a recurrent dislocation during that time. Patients with an elevated BMI or an unstable hemiarthroplasty before revision RSA are at increased risk for postoperative prosthetic dislocation. RSA is a reasonable option for treatment of prosthetic instability. To minimize recurrent instability, consideration should be given for use of larger glenospheres, increasing the lateral offset, avoiding bony impingement, and possibly using semiconstrained liners.

Acknowledgments We thank Bassem T. Elhassan MD and Scott P. Steinmann MD (both from the Department of Orthopedic Surgery, Mayo Clinic, Rochester, MN, USA) who performed the RSAs for instability.

\section{References}

1. Abdel MP, Hattrup SJ, Sperling JW, Cofield RH, Kreofsky CR, Sanchez-Sotelo J. Revision of an unstable hemiarthroplasty or anatomical total shoulder replacement using a reverse design prosthesis. Bone Joint J. 2013;95:668-672.

2. Boileau P, Melis B, Duperron D, Moineau G, Rumian AP, Han Y. Revision surgery of reverse shoulder arthroplasty. J Shoulder Elbow Surg. 2013;22:1359-1370.

3. Boileau P, Watkinson DJ, Hatzidakis AM, Balg F. Grammont reverse prosthesis: design, rationale, and biomechanics. $J$ Shoulder Elbow Surg. 2005;14(1 suppl S):147S-161S.

4. Bufquin T, Hersan A, Hubert L, Massin P. Reverse shoulder arthroplasty for the treatment of three- and four-part fractures of the proximal humerus in the elderly: a prospective review of 43 cases with a short-term follow-up. J Bone Joint Surg Br. 2007;89:516-520.

5. Cazeneuve JF, Cristofari DJ. Delta III reverse shoulder arthroplasty: radiological outcome for acute complex fractures of the proximal humerus in elderly patients. Orthop Traumatol Surg Res. 2009;95:325-329.

6. Cheung E, Willis M, Walker M, Clark R, Frankle MA. Complications in reverse total shoulder arthroplasty. J Am Acad Orthop Surg. 2011;19:439-449.

7. Cofield RH, Edgerton BC. Total shoulder arthroplasty: complications and revision surgery. Instr Course Lect. 1990;39:449462.

8. Gerber C, Pennington SD, Nyffeler RW. Reverse total shoulder arthroplasty. J Am Acad Orthop Surg. 2009;17:284-295.

9. Glanzmann MC, Kolling C, Schwyzer HK, Audige L. Conversion to hemiarthroplasty as a salvage procedure for failed reverse shoulder arthroplasty. J Shoulder Elbow Surg. 2016;25:17951802.

10. Holcomb JO, Cuff D, Petersen SA, Pupello DR, Frankle MA. Revision reverse shoulder arthroplasty for glenoid baseplate failure after primary reverse shoulder arthroplasty. J Shoulder Elbow Surg. 2009;18:717-723.

11. Muh SJ, Streit JJ, Lenarz CJ, McCrum C, Wanner JP, Shishani Y, Moraga C, Nowinski RJ, Edwards TB, Warner JJ, Walch G, Gobezie R. Resection arthroplasty for failed shoulder arthroplasty. J Shoulder Elbow Surg. 2013;22:247-252.

12. Patel DN, Young B, Onyekwelu I, Zuckerman JD, Kwon YW. Reverse total shoulder arthroplasty for failed shoulder arthroplasty. J Shoulder Elbow Surg. 2012;21:1478-1483.

13. Sanchez-Sotelo J, Sperling JW, Rowland CM, Cofield RH. Instability after shoulder arthroplasty: results of surgical treatment. J Bone Joint Surg Am. 2003;85:622-631.

14. Schairer WW, Nwachukwu BU, Lyman S, Craig EV, Gulotta LV. National utilization of reverse total shoulder arthroplasty in the United States. J Shoulder Elbow Surg. 2015;24:91-97.

15. Stephens BC, Simon P, Clark RE, Christmas KN, Stone GP, Lorenzetti AJ, Frankle MA. Revision for a failed reverse: a 12year review of a lateralized implant. J Shoulder Elbow Surg. 2016;25:e115-124.

16. Trappey GJ 4th, O'Connor DP, Edwards TB. What are the instability and infection rates after reverse shoulder arthroplasty? Clin Orthop Relat Res. 2011;469:2505-2511.

17. Wagner E, Houdek MT, Griffith T, Elhassan BT, Sanchez-Sotelo J, Sperling JW, Cofield RH. Glenoid bone-grafting in revision to a reverse total shoulder arthroplasty. J Bone Joint Surg Am. 2015;97:1653-1660.

18. Walker M, Willis MP, Brooks JP, Pupello D, Mulieri PJ, Frankle MA. The use of the reverse shoulder arthroplasty for treatment of failed total shoulder arthroplasty. J Shoulder Elbow Surg. 2012;21:514-522.

19. Werner BC, Burrus MT, Browne JA, Brockmeier SF. Superobesity (body mass index $>50 \mathrm{~kg} / \mathrm{m} 2$ ) and complications after total shoulder arthroplasty: an incremental effect of increasing body mass index. J Shoulder Elbow Surg. 2015;24:1868-1875.

20. Werner CM, Steinmann PA, Gilbart M, Gerber C. Treatment of painful pseudoparesis due to irreparable rotator cuff dysfunction with the Delta III reverse-ball-and-socket total shoulder prosthesis. J Bone Joint Surg Am. 2005;87:1476-1486. 